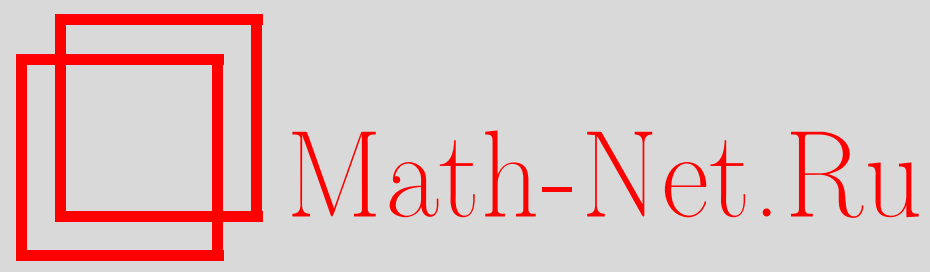

И. Г. Царьков, Неединственность решений некоторых дифференциальных уравнений и их связь с геометрической теорией приближения, Матем. заметки, 2004, том 75, выпуск 2, 287-301

DOI: https://doi.org/10.4213/mzm34

Использование Общероссийского математического портала Math-Net.Ru подразумевает, что вы прочитали и согласны с пользовательским соглашением http://www.mathnet.ru/rus/agreement

Параметры загрузки:

IP : 18.208 .226 .222

26 апреля 2023 г., $15: 19: 58$

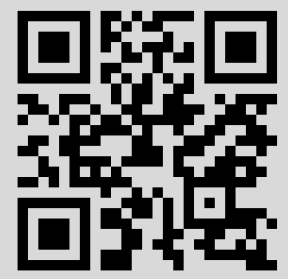




\section{НЕЕДИНСТВЕННОСТЬ РЕШЕНИЙ НЕКОТОРЫХ ДИФФЕРЕНЦИАЛЬНЫХ УРАВНЕНИЙ И ИХ СВЯЗЬ С ГЕОМЕТРИЧЕСКОЙ ТЕОРИЕЙ ПРИБЛИЖЕНИЯ}

\section{И.Г. Царьков}

В работе изучаются структурные и аппроксимативные свойства множеств, допускающих полунепрерьвную сверху ацикличную выборку из оператора почти наилучшего приближения. На основе этих свойств исследуются вопросы неединственности нелинейной неоднородной задачи Дирихле.

Библиография: 10 названий.

Введение. Через $(B)$ обозначим класс всех действительных банаховых пространств. Для банахова пространства $X$, множеств $M, N \subset X$, точки $x \in X$ определим следующие величины:

$$
\varrho(x, M)=\inf _{y \in M}\|x-y\|, \quad D(M, N)=\sup _{y \in N} \varrho(y, M), \quad d(x, M)=D(\{x\}, M) .
$$

Для произвольных числа $\varepsilon \geqslant 0$ и множества $M \subset X$ через $P_{M}^{\varepsilon}$ (соответственно $P_{M}$ ) обозначим метрический $\varepsilon$ - проектор (соответственно проектор) на множество $M$, т.е. такое отображение, что для всех $x \in X$

$$
\begin{aligned}
& P_{M}^{\varepsilon} x=\{y \in M \mid\|y-x\| \leqslant \varrho(x, M)+\varepsilon\}, \\
& P_{M} x=\{y \in M \mid\|y-x\|=\varrho(x, M)\} .
\end{aligned}
$$

ОПРЕДЕЛЕНИЕ. Элемент $x \in X \in(B)$ назьвается точкой аппроксимативной компактности множества $M \subset X$, если из любой последовательности $\left\{y_{n}\right\} \subset M$ такой, что $\left\|y_{n}-x\right\| \rightarrow \varrho(x, M), n \rightarrow \infty$, можно выделить подпоследовательность, сходящуюся к элементу этого множества. Через $A C(M)$ обозначим множество всех точек апроксимативной компактности множества $M$. При этом, если $A C(M)=X$, то множество $M$ назьвается аппроксимативно компактным.

ОПредЕЛЕниЕ. Множество $A \subset X \in(B)$ назьвается ацикличным, если $A$ непусто, компактно и группы гомологий $H_{q}, q \geqslant 1$, и приведенная группа $\widetilde{H}_{0}$ тривиальны (см. [1]). Отображение $F: M \rightarrow 2^{X}, M \subset X$, назьвается ацикличным, если для всех $x \in M$ множество $F x$ ациклично.

Работа выполнена при финансовой поддержке Российского фонда фундаментальных исследований, грант № 02-01-00248. 
ОПРЕДЕЛЕНИЕ. Множество $M \subset X \in(B)$ называется $P$-ацикличным относительно мнохсества $N \subset X$, если для всех $x \in N$ множество $P_{M} x$ ациклично. При этом, если $N=X$, то множество $M$ назьвается $P$-ацикличны.м.

ОПРЕДЕЛЕНИЕ. Отображение $F: K \rightarrow 2^{X}, K \subset X \in(B)$, назьвается полунепрерывным сверху, если для любого открытого множества $G \subset X$ множество $\{x \in K \mid$ $G \supset F x \neq \varnothing\}$ открыто в $K$. Отображение $\Psi: K \rightarrow 2^{X}$ назьвается полунепрерывной сверху ацикличной выборкой из отображения $\Phi: K \rightarrow 2^{X}, K \subset X \in(B)$, если $\Psi$ полунепрерьвно сверху и для любого $x \in K$ множество $\Psi x$ ациклично и содержится в $\Phi x$.

ОПредЕЛЕНИЕ. Пусть $M \subset X \in(B), \varepsilon \geqslant 0$. Отображение $\Psi: N \rightarrow 2^{M}$ назьвается $\varepsilon$-выборкой относительно $N$ из метрической проекиии на множество $M$, если для всех $x \in N$ множество $\Psi x \subset P_{M}^{\varepsilon} x$.

Для $x \in X \in(B), r \geqslant 0$ положим

$$
\begin{aligned}
& B(x, r)=\{y \in X \mid\|x-y\| \leqslant r\}, \\
& \stackrel{\circ}{B(x, r)}=\{y \in X \mid\|x-y\|<r\}, \\
& S(x, r)=\{y \in X \mid\|x-y\|=r\}, \quad S=S(0,1) .
\end{aligned}
$$

Через $S^{*}$ обозначим единичную сферу пространства $X^{*}$, сопряженного с $X$.

Через $(R)$ обозначим класс всех строго вьпуклых пространств, т.е. таких $X \in(B)$, что для любых различных $x, y \in S$ верно неравенство $\|(x+y) / 2\|<1$.

Через $(S S)$ обозначим класс всех сильно гладких пространств, т.е. таких $X \in(B)$, что для любой точки $x \in S$, любого функционала $x^{*} \in S^{*}$ такого, что $x^{*}(x)=1$, и любых последовательностей $\left\{x_{n}\right\} \subset X$ и $\left\{r_{n}\right\} \subset \mathbb{R}_{+}$таких, что $r_{n} \rightarrow \infty, n \rightarrow \infty$, и $x \notin B\left(x_{n}, r_{n}\right) \supset B(0,1-1 / n), n \in \mathbb{N}$, верно включение

$$
\left\{y \in X \mid x^{*}(y)<1\right\} \subset \bigcup_{n \in \mathbb{N}} B\left(x_{n}, r_{n}\right)
$$

Настоящая работа посвящена изучению структурных и аппроксимативных свойств множеств $M \subset X \in(B)$, обладающих полунепрерывной сверху ацикличной $\varepsilon$-выборкой (для любого $\varepsilon>0$ ) из метрической проекции на $M$ относительно некоторого всюду плотного вьпуклого подмножества пространства $X$. В частности, будут доказаны следуюшие утверждения.

Tеорема 1. Пусть $X \in(B), M \subset X$ - аппроксимативно компактное множес-

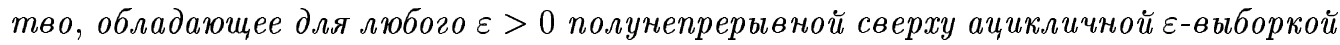
из метрической проекции на себя относительно некоторого выпуклого множества $L \subset X$, всюду плотного в $X$. Тогда множсество $M$ является $P$-ацикличным.

Teорема 2. Пусть $X \in(B)$ таково, что $X \in(S S)$ или $X^{*} \in(R)$, множсество $M \subset X$ аппроксимативно компактно и невыпукло. Тогда для любого выпуклого множества $L \subset X$, всюду плотного в $X$, найдется точка $x \in L$ такая, что мнохество $P_{м} x$ не аииклично. 
Опираясь на последнее утверждение и используя вариационньй принцип, мы выведем неединственность ненулевых решений в классе $\stackrel{\circ}{W} \underset{2}{1}(\Omega)$ в задаче Дирихле

$$
\left\{\begin{array}{l}
\Delta u=\gamma u+F(x, u)+\varphi(x), \\
u=0 \text { на } \partial \Omega
\end{array}\right.
$$

для некоторой бесконечно дифференцируемой функции $\varphi$, носитель которой содержится внутри ограниченной замкнутой области $\Omega \subset \mathbb{R}^{n}$ (см. теорему 3 ). Отметим, что впервые такого сорта задачи начали изучать Красносельский, Рутицкий, Стеценко (см. [2][4]), Похожаев (см. [5], [6]).

\section{Аппроксимативные и структурные свойства множеств неединственнос-} ти. Нам понадобятся следующие утверждения.

Теорема А (теорема Вьеториса-Бегла [7]). Пусть бикомпакт X непрерывно отображается на бикомпакт $Y$ так, что все прообразы ацикличны в размерностях $0,1, \ldots, N-1$. Тогда это отображсние индуиирует отображсение гомологических групп $H_{i}(X) \rightarrow H_{i}(Y)$, которое для $i \leqslant N-1$ является изоморфизмом, адля $i=N$ эпиморфизмом.

Из этой теоремы немедленно вытекает следующее утверждение.

Tеорема В. Пусть $X \in(B), \quad A \subset X$ - ацикличный компакт, $\Phi: A \rightarrow 2^{X}$ - полунепрерывное сверху ацикличное отображсение. Тогда мнохсество $\{(x, y) \mid$ $x \in A, y \in \Phi x\}$ ациклично в $X \times X$.

Для любых $x \in X \in(B)$ и $K \subset X, K \neq \varnothing$, через cone $\{x, K\}$ обозначим множество $\bigcup_{y \in K}[x, y]$.

Лемма 1. Пусть $X \in(B), \quad M \subset X, \quad x \in A C(M), \quad K=\operatorname{cone}\left\{x, P_{M} x\right\}$ u $\partial \Omega_{\text {g }}$ любого $\varepsilon>0$ существует полунепрерывная сверху ацикличная в-выборка из метрической проекиии на множество $M$ относительно $K$. Тогда множество $P_{M} x$ ациклично.

ДокАЗАТЕЛЬСтво. Не теряя общности, будем считать, что $x=0, \varrho(x, M)=1$. Так как $x \in A C(M)$, то $P_{M} x$ непусто и компактно. Поскольку множество $K$ звездно относительно точки $x$, то оно стягиваемо по себе в точку $x$ и, следовательно, ациклично (см. [8]). Вложим множество $P_{M} x$ в $X \times X$ как подмножество $A=\left\{(y, 0) \mid y \in P_{M} x\right\}$. Для того чтобы доказать, что множество $A$ ациклично, достаточно проверить, что любой бесконечньй цикл $\gamma$ из $A$ гомологичен нулю в $A$. Доказательство проведем от противного. Допустим, что существует бесконечный цикл $\gamma$, не гомологичный нулю в $A$. Тогда найдется окрестность $U \subset X \times X$ множества $A$, в которой цикл также не гомологичен нулю (см. [8, с. 43]). Введем на $Y=X \times X$ норму, для любой точки $(a, b) \in Y$ положив

$$
\|(a, b)\|_{Y}=\max \left\{\|a\|_{X},\|b\|_{X}\right\} .
$$

Найдется $\varepsilon \in(0,1)$ такое, что

$$
U_{\varepsilon}(A)=\{z \in Y \mid \varrho(z, A)<\varepsilon\} \subset U .
$$


Отметим, что для всех $y \in U_{\varepsilon}(A)$ верны соотношения

$$
\|y-(x, 0)\| \leqslant \varrho(y, A)+\sup _{z \in A}\|z-(x, 0)\|=\varrho(y, A)+1 \leqslant \varepsilon+1<2 .
$$

Пусть

$$
V(\eta)=\left\{z \in X \mid \varrho\left(z, P_{M} x\right)<\eta\right\}, \quad \eta>0
$$

тогда

$$
V(\varepsilon) \times \stackrel{\circ}{B}(0, \varepsilon) \subset U_{\varepsilon}(A) .
$$

Tак как $x \in A C(M)$, то найдется $\delta \in(0, \varepsilon / 4)$ такое, что $P_{M}^{\delta} x \subset V(\varepsilon)$ (см. [9, лемма 2]). В силу предположений леммы найдется полунепрерьвное сверху ацикличное отображение $\varphi: K \rightarrow 2^{M}$, являющееся $\delta$-выборкой из метрической проекции относительно $K$. Построим полунепрерывное сверху ацикличное отображение $\Psi: K \rightarrow 2^{X}$, положив

$$
\Psi(z)= \begin{cases}\varphi(z), & \text { где } z \in \alpha P_{M} x, \quad \alpha \in\left[0,1-\frac{\varepsilon}{4}\right], \\ \frac{4(\|z\|-1)+\varepsilon}{\varepsilon\|z\|} z+\frac{4(1-\|z\|)}{\varepsilon} \varphi(z), & \text { где } z \in \alpha P_{M} x, \quad \alpha \in\left(1-\frac{\varepsilon}{4}, 1\right] .\end{cases}
$$

Нетрудно видеть, что $\Psi(z)=\{z\}$ для всех $z \in P_{M} x$. По теореме В множество

$$
E_{1}=\left\{\left(z, \frac{\varepsilon}{4}(y-z)\right) \mid z \in K, y \in \Psi(z)\right\},
$$

являющееся графиком отображения $(\varepsilon / 4)(\Psi-\mathrm{Id})$, будет ацикличньм. Докажем, что множество

$$
E_{2}=\left\{\left(y, \frac{\varepsilon}{4}(y-z)\right) \mid z \in K, y \in \Psi(z)\right\}
$$

также ациклично. Это следует из того, что отображение $\zeta: E_{2} \rightarrow E_{1}$, задаваемое формулой $\zeta((a, b))=(a-4 b / \varepsilon, b)$, является гомеоморфизмом.

Кроме того, $E_{2} \supset A$. Докажем, что $E_{2} \subset U_{\varepsilon}(A)$. Действительно, во-первых, для вcex $z \in K$

$$
\varphi(z) \subset P_{M}^{\delta} z \subset P_{M}^{\delta} x \subset V(\varepsilon),
$$

и во-вторых, для всех $z \in \alpha P_{M} x, \alpha \in(1-\varepsilon / 4,1]$ и точки $t=z /\|z\| \in P_{M} x$ верны соотношения

$$
\begin{aligned}
d(t, \Psi(z)) & =d(0, \Psi(z)-t)=d\left(0, \frac{4(\|z\|-1)}{\varepsilon\|z\|} z+\frac{4(1-\|z\|)}{\varepsilon} \varphi(z)\right) \\
& =\frac{4(1-\|z\|)}{\varepsilon} d\left(0,-\frac{z}{\|z\|}+\varphi(z)\right)=\frac{4(1-\|z\|)}{\varepsilon} d(t, \varphi(z)) \leqslant d(t, \varphi(z)) \\
& \leqslant\|t-z\|+d(z, \varphi(z)) \leqslant\|t-z\|+d\left(z, P_{M}^{\delta} z\right) \leqslant\|t-z\|+\varrho(z, M)+\delta \\
& \leqslant \delta+2\|t-z\| \leqslant \delta+\frac{2 \varepsilon}{4}<\varepsilon .
\end{aligned}
$$

Отсюда следует, что $\Psi(K) \subset V(\varepsilon)$. Кроме того, для всех $z \in K$

$$
d(z, \Psi(z)) \leqslant\|x-z\|+d(x, \Psi(z)) \leqslant\|x-z\|+d(x, V(\varepsilon))<3,
$$

следовательно, $(\varepsilon / 4)(\Psi(z)-z) \subset \stackrel{\circ}{B}(0, \varepsilon)$. Тем самым, мы получаем, что $E_{2} \subset U_{\varepsilon}(A)$. Поэтому цикл $\gamma \subset A \subset E_{2} \subset U_{\varepsilon}(A) \subset U$ гомологичен нулю в $E_{2}$, и следовательно, в $U$, а это противоречит построению множества $U$. Таким образом, доказано, что $P_{M} x$ ациклично. Лемма доказана. 
Tеорема 1. Пусть $X \in(B), M \subset X$ - аппроксимативно компактное множес-

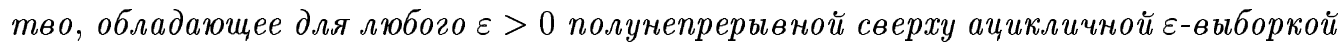
из метрической проекции на себя относительно некоторого выпуклого множества $L \subset X$, всюду плотного в $X$. Тогда множество $M$ является $P$-аиикличным.

ДокАЗАТЕльСтво. Для любого $\varepsilon>0$ найдется локально конечное покрытие пространства $X$ открытыми множествами $\left\{U_{\alpha}\right\}_{\alpha \in \mathscr{A}}$, диаметр которых меньше $\varepsilon$. Для каждого $\alpha \in \mathscr{A}$ сушествует такая функция $\varphi_{\alpha} \in C\left(X, \mathbb{R}_{+}\right)$, что $\varphi_{\alpha}>0$ на $U_{\alpha}$ и $\varphi_{\alpha} \equiv 0$ вне $U_{\alpha}$. Пусть $\psi_{\alpha}=\varphi_{\alpha} / \sum_{\alpha \in \mathscr{A}} \varphi_{\alpha}, \alpha \in \mathscr{A}$. Тогда $\psi_{\alpha} \equiv 0$ вне $U_{\alpha}, \psi_{\alpha}>0$ на $U_{\alpha}$, и $\sum_{\alpha \in \mathscr{A}} \psi_{\alpha} \equiv 1$ на $X$. Для каждого $\alpha \in \mathscr{A}$ рассмотрим некоторую точку $t_{\alpha} \in L \cap U_{\alpha}$. Построим непрерывное отображение $f(x)=\sum_{\alpha \in \mathscr{A}} t_{\alpha} \psi_{\alpha}(x)$ пространства $X$ в множество $L$. Нетрудно видеть, что для всех точек $x \in X$ верны соотношения

$$
\begin{aligned}
\|f(x)-x\| & =\left\|\sum_{\alpha: x \in U_{\alpha}} t_{\alpha} \psi_{\alpha}(x)-x\right\| \\
& =\left\|\sum_{\alpha: x \in U_{\alpha}} t_{\alpha} \psi_{\alpha}(x)-x \sum_{\alpha: x \in U_{\alpha}} \psi_{\alpha}(x)\right\| \leqslant \sum_{\alpha: x \in U_{\alpha}} \psi_{\alpha}(x)\left\|t_{\alpha}-x\right\|<\varepsilon .
\end{aligned}
$$

Положим

$$
\varphi(x)=P_{M} \circ f(x), \quad x \in X .
$$

Тогда $\varphi$ - полунепрерывное сверху ацикличное отображение. Докажем, что $\varphi(x) \subset$ $P_{M}^{\varepsilon} x$ для всех $x \in X$. Действительно,

$P_{M} \circ f(x) \subset B(f(x), \varrho(f(x), M)) \subset B(x, \varrho(f(x), M)+\|f(x)-x\|) \subset B(x, \varrho(f(x), M)+\varepsilon)$,

следовательно,

$$
\varphi(x)=P_{M} \circ f(x) \subset P_{M}^{\varepsilon} x .
$$

В силу леммы 1 для всех $x \in X$ множество $P_{M} x$ ациклично. Теорема доказана.

Teоpema C [10]. Пусть $X \in(B)$ таково, ито $X \in(S S)$ или $X^{*} \in(R), M \subset X-$ $P$-ацикличное множество с $H$-полунепрерывной сверху метрической проекиией. Тогда $M$ выпукло.

СлеДСтвие 1. Пусть $X \in(B)$ таково, что $X \in(S S)$ или $X^{*} \in(R), M \subset X-$ аппроксимативно компактное $P$-аиикличное множество. Тогда $M$ выпукло.

Teорема 2. Пусть $X \in(B)$ таково, что $X \in(S S)$ или $X^{*} \in(R)$, множество $M \subset X$ аппроксимативно компактно и невыпукло. Тогда для любого выпуклого множества $L \subset X$, всюду плотного в $X$, найдется точка $x \in L$ такая, что множество $P_{M} x$ не аииклично.

ДокАЗАТЕльСтво. Если бы для всех $x \in L$ множество $P_{M} x$ было бы ацикличным, то в силу теоремы 1 множество $M$ было бы $P$-ацикличным. Тогда в силу следствия 1 множество $M$ было бы вьпукло, чего не может быть по условию. Следовательно, верно утверждение теоремы. 
СЛЕДСТВИЕ 2. Пусть $X$ - иильбертово пространство, множество $M \subset X$ аппроксимативно компактно и невыпукло. Тогда для любого выпуклого множества $L \subset X$, всюду плотного в $X$, найдется точка $x \in L$ такая, что множество $P_{M} x$ не ациклично.

ЗАмЕчАнИЕ 1. Если пространство $X$ из теоремы 2 (следствия 2) бесконечномерно, то таких точек $x \in L$, для которых множество $P_{M} x$ не ациклично, бесконечно много. Это следует из того, что существует выпуклое подмножество $L^{\prime}$ множества $L$, всюду плотное в нем (а следовательно, и в $X$ ) и не содержащее любое наперед заданное конечное множество точек из $L$.

Неединственность в некоторых нелинейных задачах Дирихле. Пусть $\Omega \subset$ $\mathbb{R}^{n}$ - ограниченное тело, являющееся замьканием своей внутренности $\Omega_{0}$,

$$
L=\left\{f \in C^{\infty}\left(\mathbb{R}^{n}\right) \mid f \equiv 0 \text { вне } \Omega_{0}\right\} .
$$

Через $H$ обозначим гильбертово пространство, являющееся замыканием $L$ относительно евклидовой нормы

$$
\|u\|_{H}^{2} \stackrel{\text { def }}{=}\|\nabla u\|_{L_{2}}^{2}+\|\sqrt{\gamma} u\|_{L_{2}}^{2}=\int_{\mathbb{R}^{n}}(\nabla u, \nabla u) d x+\int_{\mathbb{R}^{n}} \gamma u^{2} d x,
$$

где $\gamma: \mathbb{R}^{n} \rightarrow \mathbb{R}$ - измеримая неотрищательная ограниченная функция.

Пусть $F: \mathbb{R}^{n} \times \mathbb{R} \rightarrow \mathbb{R}$ - непрерывная функция такая, что для каждого $x \in \mathbb{R}^{n}$ функция $F(x, \cdot)$ не принимает нулевых значений за исключением, быть может, дискретного подмножества $\mathbb{R}$. Будем также предполагать, что для этой функции вьполняются условия вложения, т.е. для $n=2$ найдутся константы $A, B, p, b>0$ и $a \in(0,2)$ такие, что для всех $(x, u) \in \mathbb{R}^{n+1}$

$$
|F(x, u)| \leqslant A+B|u|^{b} e^{p|u|^{a}},
$$

и для $n \geqslant 3$ найдутся константы $A, B>0$ и $\alpha \in(0,(n+2) /(n-2))$ такие, что для всех $(x, u) \in \mathbb{R}^{n+1}$

$$
|F(x, u)| \leqslant A+B|u|^{\alpha} .
$$

Везде далее будем считать, что $F(x, 0)=0$ для всех $x \in \mathbb{R}^{n}$. Положим

$$
\Phi(x, u)=\int_{0}^{u} F(x, t) d t, \quad(x, t) \in \mathbb{R}^{n+1} .
$$

Для произвольного числа $c \in \mathbb{R}$ рассмотрим множество

$$
M_{c}=\left\{f \in H \mid Q(f) \stackrel{\text { def }}{=} \int_{\mathbb{R}^{n}} \Phi(x, f(x)) d x \leqslant c\right\} .
$$

Сразу же отметим, что в силу теорем вложения множество $M_{c}$ аппроксимативно компактно и $H=\bigcup_{k \in \mathbb{R}} M_{k}$.

ПРЕДЛОЖЕНИЕ 1. Если для некоторого $x \in \Omega$ функиия $F(x, \cdot)$ не является монотонно неубывающей по второй переменной, то множество $M_{c}$ невыпукло для некоторого $c \in \mathbb{R}$. 
ДокАЗАТЕльСтво. Из условия предложения следует, что для некоторого $x \in \Omega$ функция $\Phi(x, \cdot)$ не является вьпуклой вниз по второй переменной и, следовательно, найдутся числа $u_{1}$ и $u_{2}$ такие, что

$$
\Phi\left(x, \frac{u_{1}+u_{2}}{2}\right)>\frac{1}{2}\left(\Phi\left(x, u_{1}\right)+\Phi\left(x, u_{2}\right)\right) .
$$

Без потери общности можно считать, что $\Phi\left(x, u_{1}\right)+\Phi\left(x, u_{2}\right) \neq 0$. В силу непрерывности функции $F$ найдется в окрестности точки $x$ замкнутая область $A \subset \Omega_{0}$ положительной меры $q=\mu(A)$ такая, что

$$
\Phi\left(y, \frac{u_{1}+u_{2}}{2}\right)>\frac{1}{2}\left(\Phi\left(y, u_{1}\right)+\Phi\left(y, u_{2}\right)\right)+\delta
$$

для всех $y \in A$ и некоторого $\delta \in(0,1)$. Зафиксируем некоторую функцию $\varphi \in L$. Тогда существует константа $P>1$, попарно непересекаюшиеся замкнутые множества $A_{n}^{1}, A_{n}^{2} \subset A$ такие, что

$$
\mu\left(A_{n}^{1}\right)=\mu\left(A_{n}^{2}\right)=\frac{1}{2} q-\frac{q}{4 n}
$$

(соответственно $B_{n}^{1}, B_{n}^{2} \subset A$ такие, что

$$
\left.\mu\left(B_{n}^{1}\right)=\mu\left(B_{n}^{2}\right)=\frac{2 P-\delta}{4 P} q+\frac{q \delta}{8 P n}\right)
$$

и функции $\varphi_{n}^{1}, \varphi_{n}^{2} \in L$ (соответственно $\left.\psi_{n}^{1}, \psi_{n}^{2} \in L\right), n \in \mathbb{N}$, обладаюшие следуюшими свойствами:

1) для любого $x \in \mathbb{R}^{n}$ вьполнено $\left|\varphi_{n}^{i}\right|,\left|\psi_{n}^{i}\right|<P, i=1,2, n \in \mathbb{N}$;

2) $\varphi_{n}^{i}=\psi_{n}^{i}=\varphi$ на $\mathbb{R}^{n} \backslash A, i=1,2, n \in \mathbb{N}$;

3) $\varphi_{n}^{i}=u_{i}$ (соответственно $\psi_{n}^{i}=u_{i}$ ) на множестве $A_{n}^{1}\left(\right.$ соответственно $\left.B_{n}^{1}\right)$ для всех $i=1,2$ и всех $n \in \mathbb{N}$;

4) $\varphi_{n}^{1}=u_{2}, \varphi_{n}^{2}=u_{1}$ (соответственно $\left.\psi_{n}^{1}=u_{2}, \psi_{n}^{2}=u_{1}\right)$ на множестве $A_{n}^{2}$ (соответственно $B_{n}^{2}$ ) для всех $n \in \mathbb{N}$;

5) $\psi_{n}^{i}=0$ вне $(1 / n)$-окрестности множества $B_{n}^{1} \cup B_{n}^{1} \cup\left(\mathbb{R}^{n} \backslash A\right), i=1,2, n \in \mathbb{N}$.

Из построения следует, что для $i=1,2$ верны соотношения

$$
\lim _{n \rightarrow \infty} Q\left(\varphi_{n}^{i}\right) \stackrel{\text { def }}{=} c_{0} \neq c_{0}-\frac{q \delta}{4 P}\left(\Phi\left(u_{1}\right)+\Phi\left(u_{2}\right)\right)=c_{1} \stackrel{\text { def }}{=} \lim _{n \rightarrow \infty} Q\left(\psi_{n}^{i}\right) .
$$

Следовательно, для числа $c=\left(c_{1}+c_{0}\right) / 2$ существуют $n \in \mathbb{N}$ и функции $\varphi_{i} \in\left[\varphi_{n}^{i}, \psi_{n}^{i}\right]$ такие, что $Q\left(\varphi_{i}\right)=c, i=1,2$, т.е. $\varphi_{i} \in M_{c}, i=1,2$. С другой стороны,

$$
Q\left(\frac{\varphi_{1}+\varphi_{2}}{2}\right)-\frac{1}{2}\left(Q\left(\varphi_{1}\right)+Q\left(\varphi_{2}\right)\right) \geqslant \delta q-P\left(q-\frac{2 P-\delta}{2 P} q\right)=\frac{\delta q}{2}>0,
$$

т.е. $\left(\varphi_{1}+\varphi_{2}\right) / 2 \notin M_{c}$. Отсюда следует утверждение предложения.

Для фиксированных $c \in \mathbb{R}$ и $\varphi \in H$ изучим экстремальную задачу

$$
\|u-\varphi\|_{H}^{2} \mapsto \inf _{u \in M_{c}} .
$$


Будем рассматривать случай, когда $0 \notin \partial M_{c}, \varphi \notin M_{c}$. Из апшроксимативной компактности $M_{c}$ следует, что сушествует решение этой задачи $u \in M_{c}$; при этом нетрудно видеть, что $u \in \partial M_{c}$ и, следовательно, $u \neq 0$. Поскольку функция $u$ не может принимать дискретное число существенных ненулевых значений, то $F(x, u(x)) \neq 0$ на множестве положительной меры из $\Omega$. Тогда градиент к поверхности $\partial M_{c}$ в этой точке равен $x_{u}^{*} \in H^{*}=H$, где

$$
\left(x_{u}^{*}, h\right)=\int_{\mathbb{R}^{n}} F(x, u(x)) h(x) d x,
$$

и $x_{u}^{*} \neq 0$. Из последнего замечания следует, что

$$
\partial M_{c}=\left\{f \in H \mid \int_{\mathbb{R}^{n}} \Phi(x, f(x)) d x=c\right\}
$$

(кстати, это свойство вьполняется и для случая $0 \in \partial M_{c}$, т.е. тогда, когда $c=0$, поскольку поверхность $\partial M_{c}$ содержит только одну особую точку $\left.u=0\right)$ ). Из необходимого условия экстремума следует, что для всех $h \in H$ вьполнено соотношение

$$
2 \int_{\mathbb{R}^{n}}(\nabla h, \nabla(u-\varphi)) d x+2 \int_{\mathbb{R}^{n}} \gamma(u-\varphi) d x+\lambda \int_{\mathbb{R}^{n}} F(x, u) h(x) d x=0 .
$$

При этом $\lambda=\lambda_{u}=2\|u-\varphi\| /\left\|x_{u}^{*}\right\|>0$. Тогда для обобщенных функций верно равенство

$$
-2 \int_{\mathbb{R}^{n}} \Delta(u-\varphi) d x+2 \int_{\mathbb{R}^{n}} \gamma(u-\varphi) d x+\lambda \int_{\mathbb{R}^{n}} F(x, u) h(x) d x=0,
$$

т.е. в обобщенном смысле $u$ удовлетворяет уравнению

$$
-2 \Delta(u-\varphi)+2 \gamma(u-\varphi)+\lambda F(x, u)=0,
$$

или для $\bar{\lambda}=\bar{\lambda}_{u}=\lambda / 2=\|u-\varphi\| /\left\|x_{u}^{*}\right\|-$ уравнению

$$
\Delta u-\gamma u-\bar{\lambda} F=\Delta \varphi-\gamma \varphi
$$

Лемма 2. Пусть для некоторого $с \in \mathbb{R}$ множество $M_{c}$ является невыпукльм. Тогда существует функиия $\varphi \in L$ такая, что найдутся два различных ненулевых обобщенных решения $f_{1}, f_{2} \in H$, удовлетворяющие для некоторого $\mu>0$ уравнению $\Delta u-\gamma u-\mu F(x, u)=\Delta \varphi-\gamma \varphi$.

ДокАЗАТЕЛЬСТво. Без потери общности, можно считать, что $c \neq 0$ (это следует из непрерьвности $\Phi(x, u))$. Доказательство проведем методом от противного. Допустим, что не существует двух различных ненулевых решений $f_{1}, f_{2} \in H$, удовлетворяющих для некоторого $\mu>0$ уравнению

$$
\Delta u-\gamma u-\mu F(x, u)=\Delta \varphi-\gamma \varphi
$$

Отметим, что из условия $c \neq 0$ следует, что $0 \notin \partial M_{c}$. Случаи 1) $c>0$ и 2) $c<0$ разбираются аналогично (причем случай $c<0$ имеет смысл разбирать только, если для всех $k \geqslant 0$ множество $M_{k}$ выпукло). Рассмотрим для определенности случай $c>0$. В силу следствия 2 найдется функция $\varphi \in L$, для которой множество $A$ ближайших 
точек в множестве $M_{c}$ не ациклично. В силу замечания 1 будем считать, что $\varphi \neq 0$. Тогда для любой точки $f \in A$ и

$$
\theta(f)=\bar{\lambda}_{f}=\frac{1}{2} \lambda=\frac{\|f-\varphi\|}{\left\|x_{f}^{*}\right\|}=\frac{\varrho\left(\varphi, M_{c}\right)}{\left\|x_{f}^{*}\right\|}
$$

верно равенство

$$
\Delta f-\gamma f-\bar{\lambda}_{f} F(x, f)=\Delta \varphi-\gamma \varphi
$$

Поскольку множество $A \subset \partial M_{c}$, то оно не содержит нуля, поэтому в силу предположения от противного непрерывное отображение $\theta: A \rightarrow \mathbb{R}$ инъективно на компакте $A$, т.е. $\theta$ - гомеоморфизм между $A$ и $\theta(A) \subset \mathbb{R}$. Отсюда следует, что $\theta(A)$ - неацикличное подмножество $\mathbb{R}($ см. [8]). Отсюда следует, что $\theta(A)$ несвязно и, следовательно, существуют числа $\lambda_{1}, \lambda_{2} \in \theta(A)$ такие, что $\lambda_{1}<\lambda_{2}$, и $\left(\lambda_{1}, \lambda_{2}\right) \cap \theta(A)=\varnothing$. Разобьем множество $A$ на два непересекающихся компактных подмножества

$$
A_{1}=\left\{f \in A \mid \bar{\lambda}_{f} \leqslant \lambda_{1}\right\}, \quad A_{2}=\left\{f \in A \mid \bar{\lambda}_{f} \geqslant \lambda_{2} 1\right\}
$$

Из условий леммы следует, что $H=\bigcup_{k \geqslant c} M_{k}$. Обозначим через $A^{k}$ множество всех ближайших точек в множестве $M_{k}$ для элемента $\varphi$; тогда $A^{c}=A$, и найдется число $K \in \mathbb{R}, K>c$, такое, что $\varphi \in \partial M_{K}$ и, следовательно, $A^{K}=\{\varphi\}$.

Докажем, что для всех $k_{0} \in[c, K]$ имеет место сходимость $d\left(A^{k_{0}}, A^{k}\right) \rightarrow 0$ при $k \rightarrow k_{0}$. Действительно, если бы это было не так, то существовали бы $\varepsilon>0$ и последовательности $\left\{x_{m}\right\}$ и $\left\{k_{m}\right\}$ такие, что $x_{m} \in A^{k_{m}}$ и $x_{m} \notin \mathrm{O}_{\varepsilon}\left(A^{k_{0}}\right), m \in \mathbb{N}$, и $k_{m} \rightarrow k_{0}$, $m \rightarrow \infty$. Докажем, что

$$
\left\|\varphi-x_{m}\right\|=\varrho\left(\varphi, M_{k_{m}}\right) \rightarrow \varrho\left(\varphi, M_{k_{0}}\right), \quad m \rightarrow \infty
$$

Действительно, пусть $y_{0} \in H$ - произвольная предельная точка $\left\{x_{m}\right\}$ в слабой топологии. Из теоремы вложения следует, что функционал

$$
Q(u)=\int_{\mathbb{R}^{n}} \Phi(x, u(x)) d x, \quad u \in H,
$$

слабо непрерьвен и, следовательно, $y_{0} \in \partial M_{k_{0}}$. Рассмотрим такую произвольную подпоследовательность $\left\{x_{m^{\prime}}\right\}$ (соответственно $\left\{x_{m^{\prime \prime}}\right\}$ ) последовательности $\left\{x_{m}\right\}$, что $k_{m^{\prime}}>k_{0}\left(\right.$ соответственно $\left.k_{m^{\prime \prime}}<k_{0}\right)$ для всех $m^{\prime} \in \mathbb{N}\left(\right.$ соответственно $\left.m^{\prime \prime} \in \mathbb{N}\right)$. Тогда $\left\|\varphi-x_{m^{\prime}}\right\|=\varrho\left(\varphi, M_{k_{m^{\prime}}}\right) \leqslant \varrho\left(\varphi, M_{k_{0}}\right)$, следовательно, для предельной точки $y_{0}^{\prime} \in \partial M_{k_{0}}$ последовательности $\left\{x_{m^{\prime}}\right\}$ верны соотношения

$$
\varrho\left(\varphi, M_{k_{0}}\right) \leqslant\left\|\varphi-y_{0}^{\prime}\right\| \leqslant \liminf _{m^{\prime} \rightarrow \infty}\left\|\varphi-x_{m^{\prime}}\right\| \leqslant \limsup _{m^{\prime} \rightarrow \infty}\left\|\varphi-x_{m^{\prime}}\right\| \leqslant \varrho\left(\varphi, M_{k_{0}}\right)
$$

т.е. имеет место равенство

$$
\lim _{m^{\prime} \rightarrow \infty} \varrho\left(\varphi, M_{k_{m^{\prime}}}\right)=\varrho\left(\varphi, M_{k_{0}}\right)
$$


Поскольку для любых $p, t, p<t<k_{0}$, верны включения $M_{p} \subset M_{t} \subset M_{k_{0}}$ и в окрестности любой точки $\partial M_{k_{0}}$ есть точки $u \in M_{k_{0}}$ такие, что $Q(u)<k_{0}$, то для предельной точки $y_{0}^{\prime \prime} \in \partial M_{k_{0}}$ последовательности $\left\{x_{m^{\prime \prime}}\right\}$ верны соотношения

$$
\begin{aligned}
\varrho\left(\varphi, M_{k_{0}}\right) \leqslant\left\|\varphi-y_{0}^{\prime \prime}\right\| & \leqslant \liminf _{m^{\prime \prime} \rightarrow \infty}\left\|\varphi-x_{m^{\prime \prime}}\right\|=\liminf _{m^{\prime \prime} \rightarrow \infty} \varrho\left(\varphi, M_{k_{m^{\prime \prime}}}\right) \\
& \leqslant \limsup _{m^{\prime \prime} \rightarrow \infty} \varrho\left(\varphi, M_{k_{m^{\prime \prime}}}\right) \leqslant \varrho\left(\varphi, M_{k_{0}}\right) .
\end{aligned}
$$

Отсюда вытекает, что

$$
\lim _{m^{\prime \prime} \rightarrow \infty} \varrho\left(\varphi, M_{k_{m^{\prime \prime}}}\right)=\varrho\left(\varphi, M_{k_{0}}\right)=\left\|\varphi-y_{0}^{\prime \prime}\right\|
$$

Таким образом, мы доказали, что

$$
\left\|\varphi-x_{m}\right\|=\varrho\left(\varphi, M_{k_{m}}\right) \rightarrow \varrho\left(\varphi, M_{k_{0}}\right)=\left\|\varphi-y_{0}\right\|, \quad m \rightarrow \infty
$$

Учитьвая это и то, что $y_{0}$ - предельная точка последовательности $\left\{x_{m}\right\}$ в слабой топологии, получим, что $y_{0}$ является предельной точкой этой последовательности в сильной топологии. Поэтому найдется $l \in \mathbb{N}$ такое, что $x_{l} \in \mathrm{O}_{\varepsilon}\left(A_{k_{0}}\right)$, чего не может быть. Следовательно, для всех $k_{0} \in[c, K]$

$$
d\left(A^{k_{0}}, A^{k}\right) \rightarrow 0 \quad \text { при } \quad k \rightarrow k_{0} .
$$

Положим $\varepsilon=\left(\lambda_{2}-\lambda_{1}\right) / 4>0$. Найдется $\delta \in\left(0, \lambda_{2}-\lambda_{1}\right)$ такое, что для любой точки $f \in \mathrm{O}_{\delta}\left(A_{1}\right)$ верно неравенство $\bar{\lambda}_{f}<\lambda_{1}+\varepsilon / 2$. Существует $k_{0} \in(c, K)$ такое, что для всех $k \in\left(c, k_{0}\right)$ верно включение $A \subset \mathrm{O}_{\delta}\left(A^{c}\right)$. Докажем методом от противного, что для всех достаточно близких к $c$ чисел $k$ верно $A^{k} \cap \mathrm{O}_{\delta}\left(A_{1}\right)=\varnothing$. Допустим, что существует точка $a \in A^{k} \cap \mathrm{O}_{\delta}\left(A_{1}\right)$. Тогда для полного приращения функционала $Q$ в точке $c$ верно равенство

$$
-\Delta Q=c-k=x_{a}^{*}\left(-t \frac{x_{a}^{*}}{\left\|x_{a}^{*}\right\|}\right)+o(t),
$$

где $t>0$ выбирается так, чтобы $a-t x_{a}^{*} /\left\|x_{a}^{*}\right\| \in \partial M_{c}$. Поэтому $\Delta Q /\left\|x_{a}^{*}\right\|=t+o(t)$, при этом можно считать, что $|o(t)|<\varepsilon \Delta Q /\left(4 \varrho\left(\varphi, M_{c}\right)\right)($ поскольку $o(t)=o(\Delta Q))$. Учитывая, что $\left\|\varphi-\left(a-t x_{a}^{*} /\left\|x_{a}^{*}\right\|\right)\right\| \geqslant \varrho\left(\varphi, M_{c}\right)$, получим, что

$$
\begin{aligned}
\|\varphi-a\| & \geqslant \varrho\left(\varphi, M_{c}\right)-\left\|t \frac{x_{a}^{*}}{\left\|x_{a}^{*}\right\|}\right\| \geqslant \varrho\left(\varphi, M_{c}\right)-t=\varrho\left(\varphi, M_{c}\right)-\frac{\Delta Q}{\left\|x_{a}^{*}\right\|}+o(t) \\
& \geqslant \varrho\left(\varphi, M_{c}\right)\left(1-\frac{\Delta Q}{\varrho^{2}\left(\varphi, M_{c}\right)}\left(\lambda_{1}+\frac{\varepsilon}{4}\right)-\frac{\varepsilon}{4} \frac{\Delta Q}{\varrho^{2}\left(\varphi, M_{c}\right)}\right) \\
& =\varrho\left(\varphi, M_{c}\right)\left(1-\left(\lambda_{1}+\frac{\varepsilon}{2}\right) \frac{\Delta Q}{\varrho^{2}\left(\varphi, M_{c}\right)}\right) .
\end{aligned}
$$

Аналогично, для любого элемента $f \in A_{2}$ верна формула

$$
\Delta Q(f)=x_{f}^{*}\left(t \frac{x_{f}^{*}}{\left\|x_{f}^{*}\right\|}\right)+o(t)=\left\|x_{f}^{*}\right\| t+o(t) .
$$


Поскольку векторы $x_{f}^{*}$ и $(\varphi-f)$ сонаправлены, то

$$
\left\|\varphi-\left(f+t \frac{x_{f}^{*}}{\left\|x_{f}^{*}\right\|}\right)\right\|=\|\varphi-f\|-t=\varrho\left(\varphi, M_{c}\right)-t=\varrho\left(\varphi, M_{c}\right)-\frac{\Delta Q}{\left\|x_{f}^{*}\right\|}+o(t) .
$$

Можно считать, что

$$
|o(t)|=o(\Delta Q)<\frac{\varepsilon}{4} \frac{\Delta Q}{\varrho\left(\varphi, M_{c}\right)}
$$

Тогда

$$
\begin{aligned}
\left\|\varphi-\left(f+t \frac{x_{f}^{*}}{\left\|x_{f}^{*}\right\|}\right)\right\| & \leqslant \varrho\left(\varphi, M_{c}\right)\left(1-\frac{\left(\lambda_{2}+\varepsilon / 4\right) \Delta Q}{\varrho^{2}\left(\varphi, M_{c}\right)}\right) \\
& <\varrho\left(\varphi, M_{c}\right)\left(1-\left(\lambda_{1}+\frac{\varepsilon}{2}\right) \frac{\Delta Q}{\varrho^{2}\left(\varphi, M_{c}\right)}\right) \leqslant\|\varphi-a\|,
\end{aligned}
$$

т.е. точка $a \in M_{k}$ не является ближайшей для $\varphi$. Отсюда следует, что существует некоторое $k_{1}>c$ такое, что $A^{k} \subset \mathrm{O}_{\delta}\left(A_{2}\right)$ для всех $k \in\left(c, k_{1}\right)$. И следовательно, $D\left(A_{2}, A^{k}\right) \rightarrow 0$ при $k \downarrow c$. Поэтому найдется $\tilde{k}>c$ такое, что для всех $k \in(c, \tilde{k})$ и произвольной точки $f \in A^{k}$ верно неравенство

$$
\bar{\lambda}_{f}=\frac{\|f-\varphi\|}{\left\|x_{f}^{*}\right\|}>\lambda_{1} .
$$

Отображение $\Psi:[c, K] \rightarrow 2^{H}$, задаваемое формулой $\Psi(k)=A^{k}$, полунепрерывно сверху. Поэтому существует

$$
l=\max \left\{k \in[c, K] \mid \exists f \in \Psi(k): \bar{\lambda}_{f} \geqslant \lambda_{1}\right\} .
$$

Нетрудно видеть, что $l \neq K$. При этом для всех $f \in \Psi(l)$ вьполнено $\bar{\lambda}_{f} \neq \lambda_{1}$ (иначе бы существовали два различных решения уравнения $\left.\Delta u-\gamma u-\lambda_{1} F(u)=\Delta \varphi-\gamma \varphi\right)$. Тем самым, существует $f \in \Psi(l)$ такое, что $\bar{\lambda}_{f}>\lambda_{1}$. Докажем, что найдется точка $a \in A^{l}$ такая, что $\bar{\lambda}_{a}<\lambda_{1}$. Действительно, если бы для всех $f \in A^{l}$ было верно неравенство $\bar{\lambda}_{f}>\lambda_{1}$, то в силу полунепрерьвности сверху отображения $\Psi$ множество

$$
\left\{k \in[c, K] \mid \forall f \in \Psi(k), \bar{\lambda}_{f}>\lambda_{1}\right\}
$$

было бы открыто на отрезке $[c, K]$, следовательно, существовало бы число $l_{1} \in(l, K)$, для которого вьполнялось бы следующее свойство: для всех $f \in \Psi\left(l_{1}\right)$ имеет место оценка $\bar{\lambda}_{f}>\lambda_{1}$, а это противоречит определению числа $l$. Таким образом, найдется элемент $a \in A^{l}$ такой, что $\bar{\lambda}_{a}<\lambda_{1}$. Отметим, что при рассмотрении случая 2 ) (т.е. когда $c<0$ ) множество $M_{k}$ при $k \geqslant 0$ вьпукло и, следовательно, множество ближайших точек для $\varphi$ одноточечно. Поэтому число

$$
l=\max \left\{k \in[c, K] \mid \exists f \in \Psi(k): \bar{\lambda}_{f} \geqslant \lambda_{1}\right\}
$$

не может быть больше или равно нуля, так как в этом случае, для $f$ таких, что $\{f\}=$ $\Psi(l)$, верно неравенство $\bar{\lambda}_{f}>\lambda_{1}$. Отсюда множество

$$
\left\{k \in[c, K] \mid \forall f \in \Psi(k), \bar{\lambda}_{f}>\lambda_{1}\right\}
$$


открыто на отрезке $[c, K]$, следовательно, $l$ не равно

$$
\max \left\{k \in[c, K] \mid \exists f \in \Psi(k): \bar{\lambda}_{f} \geqslant \lambda_{1}\right\}
$$

противоречие. Таким образом, $l<0$, и далее доказательство случая 2 ) не отличается от случая 1). Продолжим доказательство случая 1). Так же, как и ранее, можно доказать, что для множества

$$
A_{2}^{l}=\left\{f \in A^{l} \mid \bar{\lambda}_{f}>\lambda_{1}\right\}
$$

верно соотношение $D\left(A_{2}^{l}, A^{q}\right) \rightarrow 0, q \downarrow l$. Отсюда следует, что найдется число $q \in(l, K]$ такое, что для всех $f \in A^{q}$ выполнено $\bar{\lambda}_{f}>\lambda_{1}$, что опять же противоречит определению числа $l$. Полученное противоречие доказьвает лемму.

ТЕОрема 3. Пусть $\gamma: \mathbb{R}^{n} \rightarrow \mathbb{R}$ - неотрицательная ограниченная измеримая функиия; непрерывная функиия $F=F(x, u): \mathbb{R}^{n+1} \rightarrow \mathbb{R}$ удовлетворяет условиям вложсения, для каждого $x \in \mathbb{R}^{n}$ функиия $F(x, \cdot)$ не принимает нулевых значений за исключением, быть может, дискретного подмножества $\mathbb{R}$, и не является монотонно неубывающей по переменной $u \in \mathbb{R}$ для некоторого $x \in \Omega ;$ кроме того, для всех $x \in \mathbb{R}^{n}$ выполнено $F(x, 0)=0$. Тогда существуют бесконечно дифферениируемая функиия $g: \mathbb{R}^{n} \rightarrow \mathbb{R}$, финитная на $\Omega$, и некоторое число $\mu>0$, для которых существуют, по крайней мере, два различных ненулевых решения $g_{1}, g_{2} \in \stackrel{\circ}{W_{2}^{1}}(\Omega)$, удовлетворяющие уравнению

$$
\Delta u-\gamma u-\mu F(x, u)=g(x)
$$

ДокАЗАТЕЛЬСТВо. Поскольку функция $F$ не является монотонной для некоторого $x \in \Omega$ по переменной $u \in \mathbb{R}$, то в силу предложения 1 для некоторого $c \in \mathbb{R}$ множество $M_{c}$ невьпукло. По лемме 2 найдутся два различных ненулевых решения $f_{1}, f_{2} \in H$, удовлетворяющие для некоторого $\varphi \in L$ и $\mu>0$ уравнению

$$
\Delta u-\gamma u-\mu F(x, u)=\Delta \varphi-\gamma \varphi
$$

Положив

$$
g_{i}=\left.f_{i}\right|_{\Omega}, \quad i=1,2, \quad \text { и } \quad g=\Delta \varphi-\gamma \varphi,
$$

получим утверждение теоремы.

СлЕДСТВИЕ 3. Пусть дополнительно к условиям теоремы 3 функиия $F$ однородна по переменной и с показателем $\alpha \neq 1$. Тогда для любой ограниченной неотрицательной измеримой функиии $\gamma: \mathbb{R}^{n} \rightarrow \mathbb{R}$ существует бесконечно дифферениируемая функиия $f: \mathbb{R}^{n} \rightarrow \mathbb{R}$, финитная на $\Omega$, для которой найдутся по крайней мере два различных ненулевых решения $f_{1}, f_{2} \in \stackrel{\circ}{W_{2}^{1}}(\Omega)$, удовлетворяющ,ие уравнению

$$
\Delta u-\gamma u-F(x, u)=f(x)
$$


ДокАЗАТЕЛЬСТвО. В силу теоремы 3 существуют бесконечно дифференпируемая функция $g: \mathbb{R}^{n} \rightarrow \mathbb{R}$, финитная на $\Omega$, и некоторое число $\mu>0$, для которых существуют по крайней мере два различных ненулевых решения $g_{1}, g_{2} \in \stackrel{\circ}{W}_{2}^{1}(\Omega)$, удовлетворяющие уравнению

$$
\Delta u-\gamma u-\mu F(x, u)=g(x) .
$$

Пусть $p=\mu^{1 /(\alpha-1)}$, тогда, положив

$$
f=p g, \quad f_{i}=p g_{i}, \quad i=1,2,
$$

получим $p^{-1} \Delta f_{i}(x)-p^{-1} \gamma f_{i}(x)-\mu p^{-\alpha} F\left(x, f_{i}(x)\right)=p^{-1} f(x)$ и, следовательно,

$$
\Delta f_{i}(x)-\gamma f_{i}(x)-F\left(x, f_{i}(x)\right)=f(x), \quad i=1,2 .
$$

Отсюда вытекает утверждение следствия.

ЗАмЕЧАнИЕ 2. Если $\partial \Omega$ представляет собой подмножество нулей некоторого алгебраического многочлена (например, $\Omega$ - шар в $\mathbb{R}^{n}$ ), то в теореме 3 (соответственно в следствии 3) в качестве $g$ (соответственно $f$ ) можно взять некий многочлен, зануляющийся на гранище области $\Omega$.

ЗАМЕчАнИЕ 3. В теореме 3 (и в следствии 3) можно рассмотреть и неограниченную функцию $\gamma$, имеющую особенности только на $\partial \Omega$ (т.е. ограниченную на любом компакте из $\left.\Omega_{0}\right)$.

ЗАмЕчАниЕ 4. Если граница области $\Omega$ липшицева и функции $\gamma, F$ гёльдеровские, то решения $g_{1}, g_{2}$ соответствующего уравнения из теоремы $3\left(f_{1}, f_{2}\right.$ из следствия 3$)$ принадлежат классу $C^{2}\left(\Omega_{0}\right) \cap C(\Omega)$.

ДокАЗАТЕльство. Так же, как и в [5], можно доказать, что почти всюду имеет место равенство

$$
\begin{aligned}
g_{i}(x) & =\int_{\Omega} G(x, y)(\gamma u(y)+\lambda F(y, u(y))+g(y)) d y \\
\left(f_{i}(x)\right. & \left.=\int_{\Omega} G(x, y)(\gamma u(y)+\lambda F(y, u(y))+f(y)) d y\right), \quad i=1,2
\end{aligned}
$$

где $G(x, y)$ - функция Грина, соответствующая нулевым граничным условиям. Отсюда, из теорем вложения и свойства интегралов типа потенциала вытекает утверждение замечания.

Пусть теперь $\Omega \subset \mathbb{R}^{n}$ - ограниченная область с гладкой границей, $\tau: \Omega \rightarrow \mathbb{R}$ - бесконечно дифференцируемая функция. Пусть $F_{1}: \Omega \times \mathbb{R} \rightarrow \mathbb{R}$ - непрерьвная функция такая, что для каждого $x \in \Omega$ функция $F_{1}(x, \cdot)$ не принимает нулевых значений, за исключением, быть может, дискретного подмножества $\mathbb{R}$. Будем также предполагать, что для этой функции вьполняются условия вложения, т.е. для $n=2$ найдутся константы $A, B, p, b>0$ и $a \in(0,2)$ такие, что

$$
\forall(x, u) \in \Omega \times \mathbb{R} \quad\left|F_{1}(x, u)\right| \leqslant A+B|u|^{b} e^{p|u|^{a}},
$$


и для $n \geqslant 3$ найдутся константы $A, B>0$ и $\alpha \in(0,(n+2) /(n-2))$ такие, что

$$
\forall(x, u) \in \Omega \times \mathbb{R} \quad\left|F_{1}(x, u)\right| \leqslant A+B|u|^{\alpha} .
$$

Положим

$$
\Phi_{1}(x, u)=\int_{0}^{u} F_{1}(x, t) d t, \quad(x, t) \in \Omega \times \mathbb{R} .
$$

Пусть

$$
L_{\tau}=\left\{f \in C^{\infty}\left(\mathbb{R}^{n}\right)|f|_{\partial \Omega}=\left.\tau\right|_{\partial \Omega}\right\} .
$$

Через $H_{\tau}$ обозначим гильбертову плоскость, являющуюся замьканием $L_{\tau}$ относительно евклидовой нормы

$$
\|u\|_{H_{\tau}}^{2} \stackrel{\text { def }}{=}\|\nabla u\|_{L_{2}(\Omega)}^{2}+\|\sqrt{\gamma} u\|_{L_{2}(\Omega)}^{2}=\int_{\Omega}(\nabla u, \nabla u) d x+\int_{\Omega} \gamma u^{2} d x
$$

где $\gamma: \Omega \rightarrow \mathbb{R}$ - неотрицательная измеримая ограниченная функция. Для произвольного числа $c \in \mathbb{R}$ рассмотрим множество

$$
M_{c}^{\tau}=\left\{f \in H_{\tau} \mid Q^{\tau}(f) \stackrel{\text { def }}{=} \int_{\Omega} \Phi_{1}(x, f(x)) d x \leqslant c\right\} .
$$

Рассматривая для фиксированных $c \in \mathbb{R}$ и $\varphi \in H_{\tau}$ экстремальную задачу

$$
\|u-\varphi\|_{H_{\tau}}^{2} \mapsto \inf _{u \in M_{c}^{\tau}}
$$

аналогично теореме 3 можно доказать следующее утверждение.

ТЕОремА 4. Пусть $\Omega \subset \mathbb{R}^{n}$ - ограниченная область с гладкой граничей, $\gamma: \Omega \rightarrow$ $\mathbb{R}$ - неотрицательная ограниченная измеримая функиия, $\tau: \Omega \rightarrow \mathbb{R}$ - бесконечно дифферениируемая функиия; непрерывная функиия $F_{1}: \Omega \times \mathbb{R} \rightarrow \mathbb{R}$ удовлетворяет условиям вложения, для каждого $x \in \Omega$ функиия $F_{1}(x, \cdot)$ не принимает нулевых значений за исключением, быть может, дискретного подмножсества $\mathbb{R}$, и не является монотонно неубывающей по переменной $u \in \mathbb{R}$ для некоторого $x \in \Omega$. Тогда существуют бесконечно дифференцируемая функиия $g: \Omega \rightarrow \mathbb{R}$ такая, что $\left.g\right|_{\partial \Omega}=\left.\tau\right|_{\partial \Omega}$, и некоторое число $\mu>0$, для которых найдутся различные решения $g_{i} \in W_{2}^{1}(\Omega)$ такие, что $\left.g_{i}\right|_{\partial \Omega}=\left.\tau\right|_{\partial \Omega}, i=1,2$, удовлетворяюш, ие уравнению

$$
\Delta u-\gamma u-\mu F_{1}(x, u)=g(x) .
$$

ЗАмЕчАнИЕ 5. Если дополнительно к условиям теоремы 4 функции $\gamma$ и $F_{1}$ гёльдеровские, то решения $g_{i}, i=1,2$, принадлежат классу $C^{2}\left(\Omega_{0}\right) \cap C(\Omega)$. 


\section{СПИСОК ЦИТИРОВАННОЙ ЛИТЕРАТУРЫ}

[1] Стинрод Н., Эйленберг С. Основания алгебраической топологии. М.: Физматгиз, 1958.

[2] Красносельский М. А., Рутицкий Я. Б. Выпуклые функции и пространства Орлича. M.: 1958.

[3] Levinson N. Positive eigenfunctions for $\Delta u+\lambda f(u)=0$ // Arch. Rat. Mech. and Analysis. 1962. V. 11. № 3. P. 258-272.

[4] Красносельский М. А., Стеценко В. Я. О некоторых нелинейных задачах, имеющих много решений // Сиб. матем. ж. 1963. Т. 4. №1. С. 120-137.

[5] Похожаев С. И. О собственных функциях уравнения $\Delta u+\lambda f(u)=0 / /$ Докл. АН СССР. 1965. T. 165. №1. С. 36-39.

[6] Похожаев С. И. О одном подходе к нелинейным уравнениям // Докл. АН СССР. 1979. T. 247. №6. C. 1327-1331.

[7] Begle T. G. The Vietoris mapping theorem for bicompact spaces // Ann. of Math. 1950. V. 51. № 3. P. 534-543.

[8] Борсук К. Теория ретрактов. М.: Мир, 1971.

[9] Царьков И. Г. О связности некоторых классов множеств в банаховых пространствах // Матем. заметки. 1986. Т. 40. № 2. С. 174-196.

[10] Царьков И. Г. Свойства множеств, обладающих непрерьвной выборкой из оператора $P^{\delta}$ // Матем. заметки. 1990. Т. 48. №4. С. 122-131. 